\title{
Research on the Dynamics of Red Tide Algae Density Evolution Mechanism and Control
}

\author{
Dong-Wei HUANG ${ }^{1, a,{ }^{*}}$, Shen-Xin $\mathrm{YU}^{2, \mathrm{~b}}, \mathrm{Yu}-\mathrm{Xia} \mathrm{FU}^{3, \mathrm{c}}$ \\ ${ }^{1,2,3}$ School of science, Tianjin Polytechnic University \\ atjhuangdw@163.com, b1027616488@qq.com, 1105562138@qq.com \\ ${ }^{*}$ Corresponding author
}

Keywords: Red Tide, Chaetoceros, Routh-Hurwitz Criterion, Lyapunov-Exponents Spectrum.

\begin{abstract}
In this paper we investigate the interrelation between the concentration of nutrients and the density of Chaetoceros and algae I (algae with appropriate external replenishment), we establish mathematic model to describe the dynamic evolution behaviors between the three; The stability state of material evolutionary process in the model is analyzed and judged by Routh-Hurwitz criterion; And we select the increase rate of the concentration of nutrients as the key parameter. We could get the conditions when Hopf bifurcation occurs in the system. At the end, through numerical simulation, we calculate Lyapunov Exponent spectrum and parameter threshold when Hopf bifurcation occurs in the system. The result indicates that the concentration of nutrients play a vital role in the change of system state. And then this helps to identify the evolutionary mechanism of the system to produce the bifurcation and chaos phenomena. Finally we get the method to restrain the blooms of red tide through controlling the key parameter.
\end{abstract}

\section{Introduction}

In the ocean, Planktonic algae and protozoa died sharply after the outbreak of a large number of breeding as the characteristics of the Marine red tide phenomenon, and now the phenomenon present the increasing trend year by year, and this have influenced seriously the sustainable development of the coastal ecological environment and economy.

About the cause of red tide disaster classification, different researchers in the field of professional made a lot of beneficial explorations from different angles. Natural geographic features[1], hydrodynamic conditions and nutrient sources and transportation way in sea area laid the foundation of red tide disasters. Nutrients mainly comes from the land, atmosphere, the sea bottom sediment and Marine aquaculture on the bait is an important material basis of red tide occurred.

Algae need water to complete terrigenous nutrients, sediment and atmospheric sources of transportation work and to complete the move greatly rough the hydrodynamic condition[2]. Therefore, it plays a dual role, that is, it is not only a carrier of biogenic substance transportation, but also it is the movement of the traffic tools. Because the red tide disaster phenomena occurred in offshore, the complexity of the near sea dynamic directly leads to the complexity of the red tide genesis mechanism.

\section{Multi-Species HABs Model}

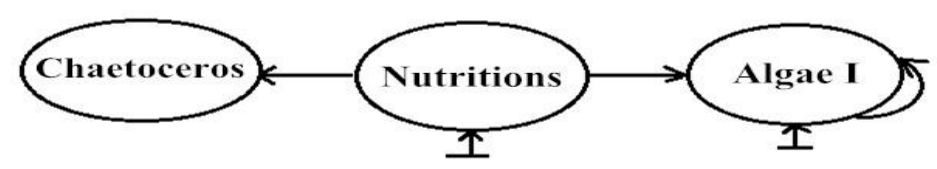

Fig. 1 Nutrition relationship system

According to the relevant literature, through the analysis of Multi-Species HABs, we can obtain the 
feedback mechanism of species food chain[3] (see Figure 1): Nutrients increased- Chaetoceros multiply rapidly-Red tide occurred, the Chaetoceros is no density dependent; Nutrients increase-Algal I(algae with appropriate external replenishment) multiply rapidly red tide occurred, In addition to the nutrients, algae I can multiply with other resources.

According to the occurrence of red tide, nutrients-Chaetoceros and nutrient-algae I can form a simple ecological system. Motivated by the discussion using principle of variety of population ecology, we construct dynamics model between nutrition, Chaetoceros and algae I as follows:

$$
\left\{\begin{array}{l}
\dot{x}=a_{10} x-m_{2} x y\left(a_{2}+x\right)^{-1}-m_{3} x z\left(a_{3}+x\right)^{-1} \\
\dot{y}=-a_{20} y+k_{2} m_{2} x y\left(a_{2}+x\right)^{-1} \\
\dot{z}=a_{30} z+k_{3} m_{3} x z\left(a_{3}+x\right)-a_{33} z^{2}
\end{array}\right.
$$

In the formula, $x=x(t), y=y(t)$ and $z=z(t)$ is respectively nutrients concentration and the density of Chaetoceros and algal I, $a_{10}$ is a quantitative input nutrient concentration; $a_{2}, a_{3}$ is the nutrient concentration growth half saturation parameters; $m_{2}, m_{3}$ and $a_{20}, a_{30}$ is respectively growth rate and mortality rate of Chaetoceros and algae $\mathrm{I} ; k_{2}$ and $k_{3}$ is the rate of absorption and conversion nutrients Chaetoceros and algae I, and $0<k_{2}, k_{3}<1$.

\section{Stability Analysis of System Model}

According to the basic characteristics of the equation, noticed the physical meaning of each element of the model and influence each other in the actual occurrence process and coupling. Consider the Lyapunov stability conditions and relevant theory of stability to judge the stability of the equation.

Equilibrium equation of the type (1), to the left is zero [4], the solution of balance is $Q\left(x^{*}, y^{*}, z^{*}\right)$. Coordinate translation, then the equation (1) can be converted into

$$
\left\{\begin{array}{l}
\dot{x}^{*}=a_{10}\left(u+x^{*}\right)-m_{2}\left(u+x^{*}\right)\left(u+y^{*}\right)\left(a_{2}+u+x^{*}\right)^{-1}-m_{3}\left(u+x^{*}\right)\left(u+z^{*}\right)\left(a_{3}+u+x^{*}\right)^{-1} \\
y^{*}=-a_{20}\left(u+y^{*}\right)+k_{2} m_{2}\left(u+x^{*}\right)\left(u+y^{*}\right)\left(a_{2}+u+x^{*}\right)^{-1} \\
z^{*}=a_{30}\left(u+z^{*}\right)+k_{3} m_{3}\left(u+x^{*}\right)\left(u+z^{*}\right)\left(a_{3}+u+x^{*}\right)^{-1}-a_{33}\left(u+z^{*}\right)^{2}
\end{array}\right.
$$

We can have the Jacobi matrix $\mathrm{K}$ of the equation (1) as follow;

$$
K=\left[\begin{array}{ccc}
a_{10}-m_{2} a_{2} y^{*}\left(a_{2}+x^{*}\right)^{-2}-m_{3} a_{3} z^{*}\left(a_{3}+x^{*}\right)^{-2} & -m_{2} x^{*}\left(a_{2}+x^{*}\right)^{-1} & -m_{3} x^{*}\left(a_{3}+x^{*}\right)^{-1} \\
-k_{2} a_{2} m_{2} y^{*}\left(a_{2}+x^{*}\right)^{-2} & -a_{20}+k_{2} m_{2} x^{*}\left(a_{2}+x^{*}\right)^{-1} & 0 \\
-k_{3} a_{3} m_{3} z^{*}\left(a_{2}+x^{*}\right)^{-2} & 0 & a_{30}+k_{3} m_{3} x^{*}\left(a_{3}+x^{*}\right)^{-1}-2 a_{33} z^{*}
\end{array}\right]
$$

According to the Lyapunov stability conditions and relevant theory of stability[5], local stability of the equation depends on the eigenvalues of Jacobi matrix K. For the three-dimensional equation, the equilibrium point should have eight kinds of cases. For the discussion in this paper equation and considering the actual situation, only four cases are considered, that is $Q_{0}\left(x_{0}, 0,0\right), Q_{1}\left(x_{1}, 0, z_{1}\right)$, $Q_{2}\left(x_{2}, y_{2}, 0\right)$ and $Q_{3}\left(x^{*}, y^{*}, z^{*}\right)$. The following discuss the local stability system under various stable state.

In equilibrium $Q_{0}\left(x_{0}, 0,0\right)$, the physical meaning is the initial level of ideal, that is, the density of Chaetoceros and algae I is zero, at this time, Jacobi matrix $K_{0}$ as follow; 


$$
K_{0}=\left[\begin{array}{ccc}
a_{10} & -m_{2} x_{0}\left(a_{2}+x_{0}\right)^{-1} & -m_{3} x_{0}\left(a_{3}+x_{0}\right)^{-1} \\
0 & -a_{20}+k_{2} m_{2} x_{0}\left(a_{2}+x_{0}\right)^{-1} & 0 \\
0 & 0 & a_{30}+k_{3} m_{3} x_{0}\left(a_{3}+x_{0}\right)^{-1}
\end{array}\right]
$$

This matrix is a triangular matrix, the three characteristic values respectively $a_{10}$, $-a_{20}+k_{2} m_{2} x_{0}\left(a_{2}+x_{0}\right)^{-1}$ and $a_{30}+k_{3} m_{3} x_{0}\left(a_{3}+x_{0}\right)^{-1}$, because of $a_{10}, a_{20}, a_{30}, k_{2}, k_{3}, a_{2}, a_{3}$ and $x_{0}$ are greater than zero. So in the three eigenvalues, $a_{10}, a_{30}+k_{3} m_{3} x_{0} /\left(a_{3}+x_{0}\right)$ must be greater than zero; therefore we can know that the solution of the system is unstable. At this condition, its practical significance is that the final result will lead to unlimited accumulation of nutrients in water and make the system unstable, only with the condition of the nutrients increased and no consumption of nutrients under no algae[6].

In equilibrium $Q_{1}\left(x_{1}, 0, z_{1}\right)$, now the density of algae $\mathrm{I}$ is not zero. Jacobi matrix $K_{1}$ for the time as follow:

$$
K_{1}=\left[\begin{array}{ccc}
a_{10}-m_{3} a_{3} z_{1}\left(a_{3}+x_{1}\right)^{-2} & -m_{2} x_{1}\left(a_{2}+x_{1}\right)^{-1} & -m_{3} x_{1}\left(a_{3}+x_{1}\right)^{-1} \\
0 & -a_{20}+k_{2} m_{2} x_{1}\left(a_{2}+x_{1}\right)^{-1} & 0 \\
-k_{3} m_{3} a_{3} z_{1}\left(a_{3}+x_{1}\right)^{-2} & 0 & a_{30}+k_{3} m_{3} x_{1}\left(a_{3}+x_{1}\right)^{-1}-2 a_{33} z_{1}
\end{array}\right]
$$

Here we use the Routh-Hurwitz criterion for identification of the stability of the system. According to Routh-Hurwitz criterion [7].

Characteristic equation of the system in point $Q_{1}\left(x_{1}, 0, z_{1}\right)$ is given by

$$
\lambda^{3}+\left(\omega_{0}+\omega_{1}+\omega_{2}\right) \lambda^{2}+\left[\omega_{0} \omega_{1}+\omega_{1} \omega_{2}+\omega_{0} \omega_{2}+\omega_{3}\right] \lambda+\left(\omega_{0} \omega_{1} \omega_{2}+\omega_{0} \omega_{3}\right)=0
$$

Where

$$
\begin{aligned}
& \omega_{0}=a_{20}-k_{2} m_{2} x_{1}\left(a_{2}+x_{1}\right)^{-1}, \omega_{1}=-a_{10}+m_{3} a_{3} z_{1}\left(a_{3}+x_{1}\right)^{-2}, \omega_{2}=-a_{30}-k_{3} m_{3} x_{1}\left(a_{3}+x_{1}\right)^{-1}+2 a_{33} z_{1} \\
& \omega_{3}=-a_{3} k_{3} m_{3}{ }^{2} x_{1} z_{1}\left(a_{3}+x_{2}\right)^{-3} .
\end{aligned}
$$

According to the Routh-Hurwitz criterion, the stability conditions of the system at the point is to make the following inequality condition $(\mathrm{R})$ established

$$
(R):\left\{\begin{array}{l}
\omega_{0}+\omega_{1}+\omega_{2}>0, \omega_{0} \omega_{1}+\omega_{1} \omega_{2}+\omega_{0} \omega_{2}+\omega_{3}>0, \quad \omega_{0} \omega_{1} \omega_{2}+\omega_{0} \omega_{3}>0 \\
\left(\omega_{0}+\omega_{1}+\omega_{2}\right)\left(\omega_{0} \omega_{1}+\omega_{1} \omega_{2}+\omega_{0} \omega_{2}+\omega_{3}\right)-\left(\omega_{0} \omega_{1} \omega_{2}+\omega_{0} \omega_{3}\right)>0
\end{array}\right.
$$

The stable state will appears transcritical bifurcation and supercritical bifurcation phenomenon with the change of parameters.

For equilibrium $Q_{2}\left(x_{2}, y_{2}, 0\right)$, the density of Chaetoceros is not zero at this time. By the Jacobi matrix, we can know that there will be a characteristic root for $\lambda_{1}=a_{30}+k_{3} m_{3} x_{2}\left(a_{3}+x_{2}\right)^{-1}$ and two other characteristic roots depend on the following quadratic equation

$$
\left(\lambda-a_{10}+m_{3} a_{3} z_{1}\left(a_{3}+x_{1}\right)^{-2}\right)\left(\lambda+a_{20}-k_{2} m_{2} x_{2}\left(a_{2}+x_{2}\right)^{-1}\right)-k_{2} a_{2} m_{2}^{2} x_{2} y_{2}\left(a_{2}+x_{2}\right)^{-3}=0
$$

The characteristic root $\lambda_{1}=a_{30}+k_{3} m_{3} x_{2}\left(a_{3}+x_{2}\right)^{-1}>0$ is permanent establishment because $a_{30}, a_{3}, k_{3}, m_{3}, x_{2}$ are all non-negative; Therefore, whether the other two eigenvalue is positive or negative, the system is not stable in the near equilibrium, At this state, there is no competition between species only Chaetoceros algae, which does not exist the living space and nutrient competition, so Chaetoceros number will make a large increase in short time when 
Chaetoceros breeds to a certain scale, causing red tide outbreak and forming red tide phenomenon.

When $y \neq 0, z \neq 0$, in equilibrium $Q_{3}\left(x^{*}, y^{*}, z^{*}\right)$, we can get $y^{*}=\left(a_{10}-m_{3} z^{*}\left(a_{3}+x^{*}\right)^{-1}\right)\left(a_{2}+x^{*}\right) m_{2}^{-1}$, $x^{*}=a_{20} a_{2}\left(k_{2} m_{2}-a_{20}\right)^{-1}, z^{*}=a_{30} a_{33}^{-1}+k_{3} m_{3} a_{33} x^{*}\left(a_{3}+x^{*}\right)^{-1}$; In ecology to make equilibrium state stable, we should let $x^{*}>0$, that is, the presence of nutrients is necessary and algae could survive. If $x^{*}<0$, in the ecology this is not an actual state. From $x^{*}>0$, we have $a_{2}<k_{2} m_{2}$

The above can be considered as the necessary condition for red tide occurrence and development.

The condition of $y^{*}>0$ is if and only if $z^{*}<\left(a_{10} a_{3}+a_{10} x^{*}\right) / m_{3}$, further $\varphi>0$

$$
\varphi=a_{10} a_{2}^{2} a_{20}^{2} a_{33}\left(k_{2} m_{2}-a_{20}\right)^{-2}+a_{2} a_{20}\left(2 a_{10} a_{3} a_{33}-m_{3}\left(a_{30}+k_{3}\right)\right)\left(k_{2} m_{2}-a_{20}\right)^{-1}+a_{3}\left(a_{10} a_{3} a_{33}-a_{30} m_{3}\right)
$$

For the system, if there is a state of balance in the equilibrium state $Q_{3}\left(x^{*}, y^{*}, z^{*}\right)$ and $x^{*}, y^{*}, z^{*}>0$, it is only in the first quadrant and the system will not produce cross bifurcation in the quadrant[8], namely saddle point.

From the analysis, equilibrium point $Q_{1}\left(x_{1}, 0, z_{1}\right)$ and $Q_{3}\left(x^{*}, y^{*}, z^{*}\right)$ appear overlap in the critical bifurcation point, at this time, $a_{2}<k_{2} m_{2}, \varphi=0$. Local bifurcation behavior at the critical point can be summarized as follows:

1) $a_{20}<k_{2} m_{2}, \varphi<0, Q_{1}\left(x_{1}, 0, z_{1}\right)$ is stable under the condition $(\mathrm{R}), Q_{3}\left(x^{*}, y^{*}, z^{*}\right)$ is the ecological non $\operatorname{practical}_{\left(y^{*}<0\right)}$

2) $a_{20}<k_{2} m_{2}, \varphi>0, Q_{1}\left(x_{1}, 0, z_{1}\right)$ is unstable, $Q_{3}\left(x^{*}, y^{*}, z^{*}\right)$ is the ecological practical and stable;

To sum up, in theory, the stability of solution of the system is conditional, and the equilibrium between different stability conditions are also different, there are overlapping phenomenon between different equilibrium.

\section{Numerical Simulation}

Considering the actual situation of red tide and the key parameters affecting the food chain model, we take $a_{10}$ as a control variable and using bifurcation theory to study the stability of system. Taken parameters $a_{20}=0.01325, a_{30}=0.16, a_{33}=0.36, m_{2}=6, m_{3}=4, k_{2}=0.08, k_{3}=0.065, a_{2}=15, a_{3}=10$. At this time, the parameters satisfied $a_{20}<k_{2} m_{2}$, the initial condition of each variable as follows: $x(0)=1, y(0)=0.3, z(0)=0.1$; We can get the numerical simulation by the parameters.

Through calculating Lyapunov Exponent of the system(see Figure 2), we know that, the largest Lyapunov Exponent of the system changes with the change of $a_{10}$. So the stability of system also changes with the change of $a_{10}$; When $a_{10}<0.47$, we know the system is stable because of the largest Lyapunov Exponent of the system blow zero; When $a_{10} \geq 0.47$, we know the largest Lyapunov Exponent of the system is more than zero so that the system is unstable; And we found the largest Lyapunov Exponent of the system occur sudden increase when $a_{10} \geq 0.53$.

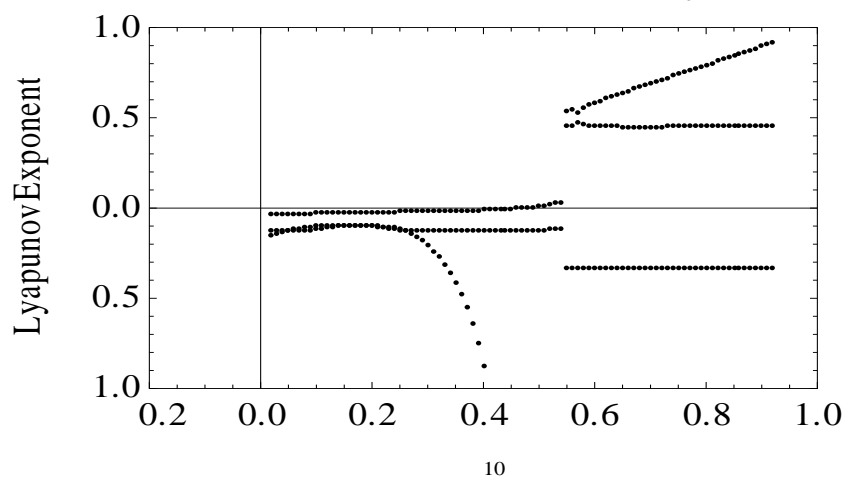

Fig. 2 Lyapunov Exponent spectrum of system 


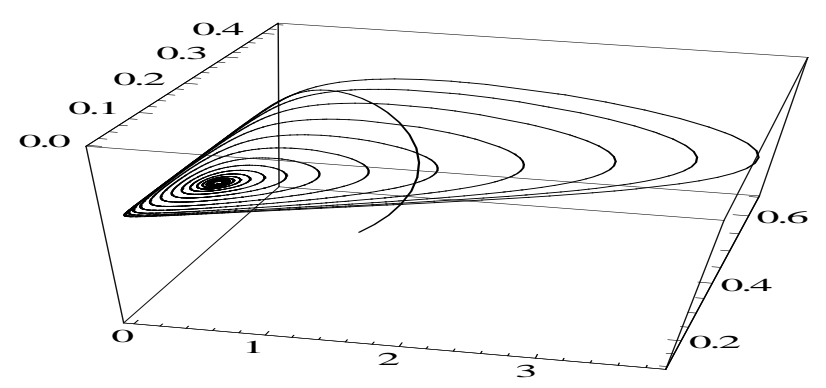

Fig. 3 When $a_{10}=0.219$,phase diagram of system

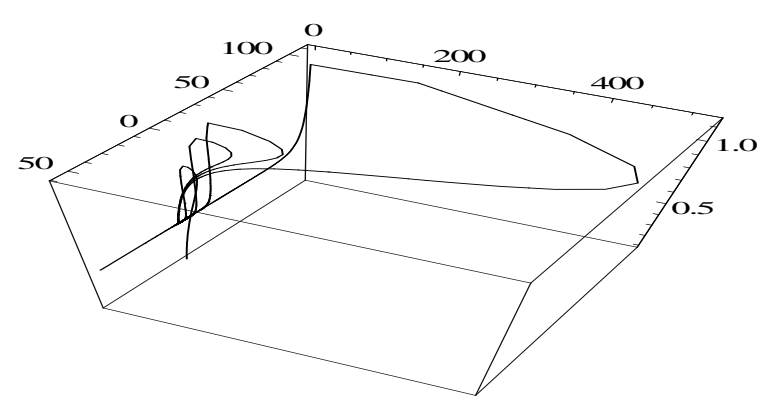

Fig. 4 When $a_{10}=0.47$, phase diagram of system

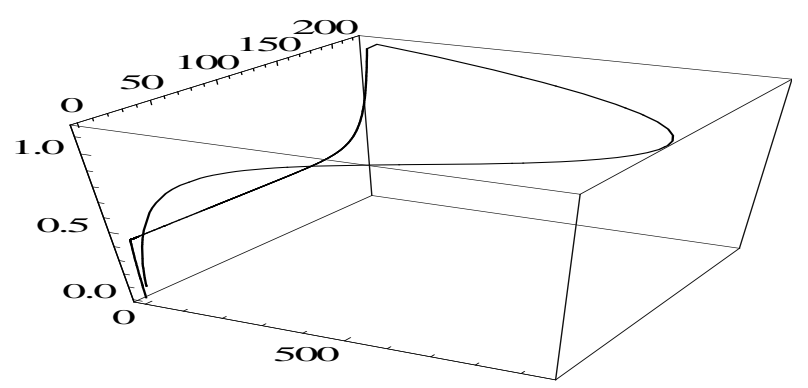

Fig. 5 When $a_{10}=0.56$, phase diagram of system

In order to verify our results, we choose control variable $a_{10}=0.219$, at this time, $\varphi=1.78649>0$; Through numerical simulation of the model, we could get phase diagram; see Figure 3; Through observing Figure 3, we conclude the result of numerical simulation which indicate nutrient concentration, the density of Chaetoceros and algae I are periodically occurring with time. When control variable $a_{10}=0.219$, the period $t$ is 400 .

In the early stage of the system, the concentration of nutrients will continue to increase with time changing because of low nutrient consumption; With the development of time, through the feedback of the system, the density of Chaetoceros will increase. And the density of algae I start to growth in the initial time. When the density of the algae I reaches a certain number, it will converge to a stable state and only appears minor fluctuations with time changing. Due to the strong dependence on nutrients and no external supply and no space constraints, the density of Chaetoceros will increase sharply after a period of time the concentration of nutrients increasing. And the growth of Chaetoceros and Algal I and nutrient concentration form a feedback mechanism, at the same time, the volume of them is always controlled within a certain range. The system is in a relatively stable state, the growth of algal is to the ecological system made no harm.

Through the numerical simulation, characteristic equations are obtained for

$$
\lambda^{3}+0.162167 \lambda^{2}+0.000888742 \lambda+0.0000817021=0
$$

According to the Routh-Hurwitz criterion, because each coefficient of $\lambda$ is positive and $\Delta_{2}=0.0000624221>0$, therefore, the system is stable at this time; Although the density of algae will 
increase and multiply with time changing, it always maintained in a certain range.

We could obtain Lyapunov Exponent of the system (-0. 0205043, -0. 125759, -0. 211796) through numerical calculation, we also know that the system is stable at this point.

When $a_{10}=0.47$ or $a_{10}=0.56$, we could obtain phase diagram through numerical simulation of the model; as shown in Figure 4 and Figure 5.

Compared to Figure 3, from Figure 4 the system now is not stable; the growth speed of concentration of nutrients will be faster, reaching a more concentration than before, the density of Chaetoceros also increased to greater $(y>5)$, at this time, the bloom of algal caused a certain extent red tide and harm to the ecological system. Compared to Figure 4, from Figure 5 we see that the system is unstable; Red tide outbreaks will become faster, the extent of the outbreak is more violent. When the concentration of nutrients is too large $(x>10, t>7)$, the density of Chaetoceros will sharply increased, and the density of Algal I will also increase considerably. From the above analysis, we obtain that the system stability is constantly with the change of $a_{10}$ : When $a_{10}<0.47$, the system is stable; When $0.47 \leq a_{10}<0.53$, the system is not stable; When $a_{10} \geq 0.53$, the system appears critical bifurcation phenomenon.

\section{Summary}

The results show that there is Hopf bifurcation phenomenon for a variety of ecological dynamics model equation. The stable state in the system is conditional, and actual conditions are identical with red tide parameters by and large. The results contribute to understanding better of dynamic behavior of the red tide species and the establishment of a more effective forecasting model of red tide.

\section{Acknowledgement}

The work reported in this article is supported by the National Natural Science Foundation of China (No.11272229 and No.11102132) and Tianjin Polytechnic University Innovation Plan for Graduate Education (No.14130).

\section{References}

[1] Zhao Dongzhi,Zhao Ling,Zhang Fengshou.Type of Formation, Distribution and temporal trend of red tides occurred in the China Sea [J] . Marine Environmental Science. 2003, 22 (3) , 8-11.

[2] Edwards AM, etal. Generic dynamics of a simple plankton population model with a non-integer exponent of closure Chaos Solitons and Fractals, 2001, 12 (2001):289-300.

[3] Wang Hongli Feng Jianfen g Shen Fei.Nonlinear Dynamics Research of the Algal Model in Bohai Sea [J] . Ocean Technology.2002, 21(3), 8-12.

[4] Wang Hong-li, Feng Jian-feng, Li Chao,Research on Nonlinear Dynamics of Multi-Species HABs Model [J] . Journal of Tianjin University, 2003, 36(4), 400-403.

[5] Chen J B, LiJ.Dynamic response and reliability analysis of nonlinear stoehastie struetures. Probabilistic Engineering Meehanies, 2005, 20(1):33-44.

[6] Appelo CAJ. Cation and proton exchange, $\mathrm{pH}$ variations, and carbonate reaction in a freshening aquifer, Water Resources Research, 1994, 30:2793-2805.

[7] Appelo CAJ. Some calculations on multi-component transport with cation exchange in aquifers Ground Water, 1994b, 32:968-975.

[8] G. F. Webb, M. J. Blaser, H. Zhu, S. Ardal and J.Wu, Critical Role of Nosocomial Transmission in the Toronto SARS treak, Mathematical Biosciences and Engineering,2004,1(1),1-13. 